\title{
Emergence of fusarioses in a university hospital in Turkey during a 20-year period
}

\author{
B. Dalyan Cilo ${ }^{1}$ - A. M. S. Al-Hatmi ${ }^{2,3,4}$ - S. Seyedmousavi ${ }^{10,11,12}$ - A. J. M. M. Rijs ${ }^{10}$ •

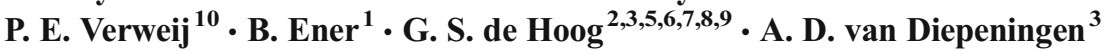

Received: 26 March 2015 / Accepted: 5 May 2015

(C) Springer-Verlag Berlin Heidelberg 2015

\begin{abstract}
Fusarium species have started appearing increasingly as the main cause of infections, particularly in immunocompromised patients. In this study, we aimed to present the first epidemiological data from Turkey, analyze fusariosis cases that have been monitored in a university hospital during the past 20 years, identify the responsible Fusarium species, and determine antifungal susceptibilities. A total of 47 cases of fusariosis was included in the study. Fusarium isolates were identified by multilocus sequence typing (MLST). Antifungal susceptibility was tested by the broth microdilution method according to the Clinical and Laboratory Standards Institute (CLSI) methodology. Of the Fusarium infections, $23.4 \%$ were superficial, $44.7 \%$ were locally invasive, and $31.9 \%$ were disseminated. A significant increase was observed over the years. The Fusarium fujikuroi
\end{abstract}

Electronic supplementary material The online version of this article (doi:10.1007/s10096-015-2405-y) contains supplementary material, which is available to authorized users.

A. D. van Diepeningen

a.diepeningen@cbs.knaw.nl

1 Department of Medical Microbiology, Uludağ University Faculty of Medicine, Bursa, Turkey

2 Institute of Biodiversity and Ecosystem Dynamics, University of Amsterdam, Amsterdam, The Netherlands

3 CBS-KNAW Fungal Biodiversity Centre, Uppsalalaan 8, 3584 CT Utrecht, The Netherlands

4 Directorate General of Health Services, Ibri Hospital, Ministry of Health, Muscat, Oman

5 Peking University Health Science Center, Research Center for Medical Mycology, Beijing, China species complex (FFSC) proved to be the most frequent agent group (17 cases; $51.5 \%$ ), followed by the Fusarium solani species complex (FSSC) (14 cases; $42.4 \%$ ), the Fusarium dimerum species complex (FDSC), and the Fusarium oxysporum species complexes (FOSC) (one case each). Amphotericin B had the highest in vitro activity against all species. Voriconazole and posaconazole showed interspecies variability across and within Fusarium species complexes. In conclusion, our data support the fact that regional differences exist in the distribution of the Fusarium species and that species-specific differences are observed in antifungal susceptibility patterns. The monitoring of local epidemiological data by determining fungal identity and susceptibility are of importance in guiding the clinical followup of patients.
6 Sun Yat-Sen Memorial Hospital, Sun Yat-Sen University, Guangzhou, China

7 Shanghai Institute of Medical Mycology, Changzheng Hospital, Second Military Medical University, Shanghai, China

8 Basic Pathology Department, Federal University of Paraná State, Curitiba, Paraná, Brazil

9 King Abdulaziz University, Jeddah, Saudi Arabia

10 Department of Medical Microbiology, Radboud University Nijmegen Medical Centre, Nijmegen, The Netherlands

11 Department of Medical Microbiology and Infectious Diseases, Erasmus MC, Rotterdam, The Netherlands

12 Invasive Fungi Research Center, Mazandaran University of Medical Sciences, Sari, Iran 


\section{Introduction}

The large genus Fusarium contains mainly species with a saprobic or plant-pathogenic lifestyle, but also contains species infamous for their adverse health effects: some have mycotoxigenic properties and/or have the ability to cause opportunistic infections in humans and animals [1]. Most of the former Fusarium species are now known to be species complexes composed of clusters of cryptic species with little to no morphological differences. Multilocus sequence typing (MLST) is necessary to distinguish between such species [2-5]. Now that the "one fungus = one name" rule has been adopted, a proposal was made to conserve the name Fusarium above linked sexual state names like Haemonectria and Gibberella [6], and a plea to keep the taxonomy of clinically important species as stable as possible [7]. In line with these proposals, we maintain a concept of the genus Fusarium in the broadest sense according to Geiser et al. [6].

Most of the opportunistic Fusarium pathogens identified in the literature are members of the $F$. solani species complex (FSSC), the F. oxysporum species complex (FOSC), and the F. fujikuroi species complex (FFSC), and less often of the F. incarnatum-equiseti species complex (FIESC), the F. dimerum species complex (FDSC), and the F. chlamydosporum species complex (FCSC) $[5,8,9]$. Some species and haplotypes causing infections have a worldwide distribution, while local variation also exists [10].

Prevalent fusariosis in otherwise healthy individuals mostly concern onychomycosis, skin infections, and keratitis, and an occasional mycetoma, especially in areas with warmer, drier climates [11]. Deep and disseminated infections are confined to severely immunocompromised patients. Disseminated infections seem predominantly related to hematological disorders [12], sometimes with a link to a pre-existing nail or cutaneous infection $[13,14]$. The observed number of fusarioses has been reported to increase over the past several decades: this may be partly due to the growing number of immunocompromised patients and their prolonged average survival time, partly due to prophylactic therapeutic approaches against opportunistic fungal infections which have little impact on Fusarium, and partly to our improved ability to identify the infective agents $[14,15]$.

Most opportunistic Fusarium species are relatively to very resistant to antifungal drugs in vitro, although anti-fungal sensitivity profiles may differ between species [2, 16, 17]. While refractory onychomycoses are not life-threatening, timely and effective treatment for deep and disseminated fusariosis is very important. Joint guidelines on the treatment of hyalohyphomycoses recommend amphotericin B and voriconazole for the treatment of systemic fusariosis [18], despite variable minimum inhibitory concentration (MIC) test results for both drugs $[2,16,17,19]$. Hence, accurate identification of the species involved is of importance for epidemiological studies and for guiding clinical follow-up of the patients $[2,13]$.

In this study, cases of fusariosis acquired in a tertiary-care university hospital in Turkey over the past 20 years were analyzed. All preserved Fusarium isolates were identified using an MLST approach suitable for the state-of-the-art identification of species complexes, species, and haplotypes, resulting in an overview of the Fusarium species occurring as etiological agents of disease. Antifungal susceptibility profiles were made for all these isolates to help clinicians develop appropriate therapies that have a high probability of successfully treating fusarioses.

\section{Materials and methods}

\section{Setting and epidemiological data}

Uludağ University Healthcare and Research Hospital is an 800-bed tertiary-care teaching institution in Bursa, Turkey. There are seven adult (Reanimation, Thoracic-Heart-Vascular Surgery, Cardiology, Plastic Surgery and Burns Unit, Neurology, Brain Surgery, and Lung Diseases) and two pediatric (Neonatal and Pediatric) intensive care units, as well as hematology and oncology wards and kidney, liver, and bone marrow transplantation units. The mycology unit of the medical microbiology laboratory receives specimens from patients admitted to the hospital and all fusariosis data regarding diagnosis and sites of infection were obtained retrospectively from laboratory records from 1 June 1995 till 31 December 2014. All cases were classified as superficial, locally invasive, or disseminated infections. The microscopic appearance of hypha and recovery of Fusarium spp. from specimens like skin, nails, and corneal scrapings were considered superficial infections. Disseminated disease was documented either by at least two positive blood cultures, or one positive blood culture together with skin or another organ involvement proven by culture and microscopy. Locally invasive pulmonary, sinonasal, and skin fusarioses were considered when Fusarium species were isolated from the respiratory tract, sinuses, or skin biopsies in the presence of risk factors, clinical signs, and symptoms, and when hyphae were seen at microscopy.

\section{Isolates}

Fusarium isolates have been collected and deposited in $10 \%$ glycerol at $-80{ }^{\circ} \mathrm{C}$ in the culture collection of Uludağ University Healthcare and Research Hospital from 1 June 1995 to 31 December 2014. The initial identification as Fusarium species was based on macroscopic and microscopic morphological features. Isolates which were able to grow after subculturing 
twice on Sabouraud dextrose agar (SDA) were also deposited in the reference collection of the CBS-KNAW Fungal Biodiversity Centre in Utrecht, the Netherlands, and these were characterized based on MLST.

\section{DNA extraction and sequencing}

The DNA of strains were extracted with the CTAB-based method of Möller et al. [20]. Tentative identification of species (complexes) was by nuclear ribosomal internal transcribed spacer (ITS) sequencing with primers ITS1 and ITS4 [21] and a part of the translation elongation factor 1-alpha (tef$1 \alpha)$ gene with primers EF1 and EF2 [22]. To determine the exact haplotypes within species, partial sequences of the rpb2 gene [5], the intergenic spacer region IGS [3], the large ribosomal subunit LSU, and the beta-tubulin gene [23] were obtained as needed. For sequence typing of members of the FSSC, LSU and rpb2 [2] were used in addition to ITS and tef- $1 \alpha$, for members of the FDSC and the FFSC, $\operatorname{rpb} 2$ and $\beta$ tubulin [23], and for the FOSC, IGS and rpb2 [3]. All strains could, thus, unambiguously be assigned to the species or haplotype level.

All polymerase chain reaction (PCR) assays were done with Bioline Taq Polymerase in 12.5- $\mu$ l volumes. Amplicons were purified with Sephadex G-50 Fine (GE Healthcare, Uppsala, Sweden), then subjected to direct sequencing with the ABI PRISM BigDye Terminator Cycle Sequencing Kit (Applied Biosystems, Foster City, CA, USA), and analyzed on an ABI PRISM 3730xl sequencer. Sequences were edited using SeqMan in the Lasergene software (DNASTAR, Madison, WI, USA).

\section{Identification}

Strains were identified by BLAST identification of the obtained sequences in GenBank, the CBS database, the Fusarium ID [22] and Fusarium MLST [5] databases. Besides phylogenetic placements with species/haplotypes within species, complexes were checked with the above mentioned available databases specific for the found species complexes.

\section{Antifungal susceptibility testing}

Isolates were tested for in vitro susceptibility to fluconazole (FLC; Pfizer Central Research Sandwich, Tadworth, Surrey, UK), itraconazole (ITC: Janssen Research Foundation, Beerse, Belgium), posaconazole (POS: Merck, Whitehouse Station, NJ, USA), voriconazole (VOR: Pfizer Central Research, Sandwich, Tadworth, Surrey, UK), amphotericin B (AMB; Bristol-Myers Squibb, Woerden, the Netherlands), caspofungin (CAS; Merck Sharp \& Dohme BV, Haarlem, the Netherlands), and anidulafungin (AND; Pfizer Central Research, Sandwich, Tadworth, Surrey, UK) by the broth microdilution method according to the Clinical and Laboratory Standards Institute (CLSI) methodology [24]. The MICs of amphotericin B, flucytosine, fluconazole, itraconazole, voriconazole, and posaconazole were determined visually: an inverted mirror was used for comparing the growth in wells containing the drugs with that in the drug-free control well. The results were also read using a microtitration plate spectrophotometric reader (Anthos htIII; Anthos Labtec Instruments, Salzburg, Austria). The minimum effective concentrations (MECs) of anidulafungin and caspofungin were read using a plate microscope (Olympus SZX9; Olympus Nederland, Zoeterwoude, the Netherlands), at $25 \times$ to $50 \times$ magnification. Paecilomyces variotii (ATCC 22319), Candida parapsilosis (ATCC 22019), and C. krusei (ATCC 6258) were used for quality controls in all experiments. The ranges and geometric means (GMs) of the MICs and MECs were determined for each species and drug after $48 \mathrm{~h}$ of incubation. If no growth was observed or the growth was not adequate, the incubation was extended to $72 \mathrm{~h}$. All experiments on each strain were performed using three independent replicates on different days.

\section{Statistical analysis}

Differences between years and MIC/MEC distributions between the groups were compared using the Chi-square test; differences were considered statistically significant at a $p$-value of $\leq 0.05$.

\section{Results}

We identified 47 cases of Fusarium infection from 1995 to 2015: 11 (23.4\%) were superficial, 21 (44.7\%) were locally invasive, and 15 (31.9\%) were disseminated. A significant increase in the number of fusarioses in 5-year intervals was observed over the past several years $(p<0.001$; Fig 1$)$. Patient data on underlying disorders are provided in Table 1. Disseminated infections were mainly associated with hematological malignancies ( $80 \% ; 12$ out of 15 cases). In the remaining patients with disseminated fusariosis, two were severely immunosuppressed due to malign melanoma and Wegener's granulomatosis, respectively, and a third was a low-birthweight newborn (Table 1).

Local invasive infections $(21 / 47 ; 44.7 \%)$ were seen in patients with diverse underlying disease conditions (Table 1). Fusarium spp. grew from respiratory tract specimens in ten patients who suffered from hematological malignancy $(n=1)$, solid organ carcinoma $(n=3)$, and various chronic diseases $(n=6)$. Local sinonasal infections, proven by nasal biopsy samples with positive microscopy and culture, were seen in five patients, four of whom had hematological malignancies and one had aplastic anemia. In six patients, Fusarium was grown from skin biopsy samples with no 


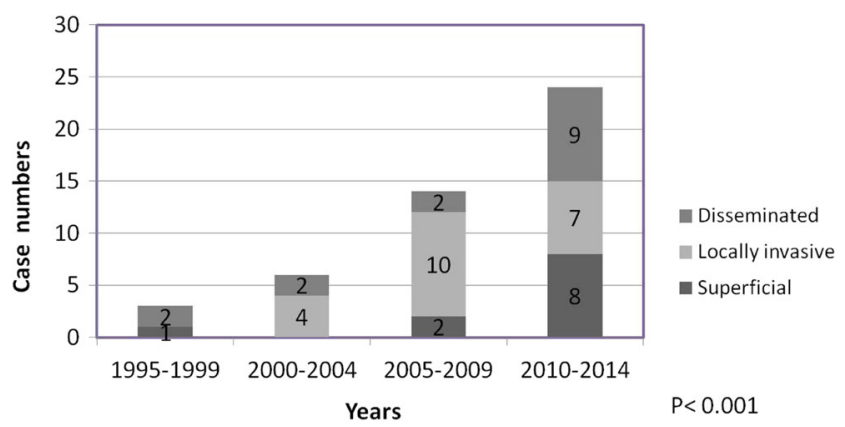

Fig. 1 Increasing incidence of Fusarium infections over the past 20 years in the studied university hospital in Turkey

growth in blood cultures. Two of these patients had a hematological malignancy, while one had solid carcinoma. Fusarium isolates were repeatedly cultured from skin biopsies of lower extremity lesions in a paraplegic patient [25]. Of the last two patients, one had chronic renal failure, while the other had hydatid cysts.

The main superficial infections were keratitis and onychomycosis ( $72.7 \%$; 8 out of 11$)$. In two chronically ill patients and one patient suffering from burn wounds, Fusarium growth was obtained from skin scrapings.

MLST was performed on 46 revivable isolates from 33 of the 47 patients $(70.2 \%$ ) described above (Table 2$)$. The FFSC proved to be the most frequent agent group (17 cases; $51.5 \%$ ), followed by the FSSC (14 cases; $42.4 \%$ ), and the FDSC and the FOSC (one case each). The distribution of the isolated species differed among the patients with various clinical presentations. Members of the FFSC were the most frequent etiological agents of disseminated infections $(8 / 12 ; 66.7 \%)$, followed by Fusarium petroliphilum of the FSSC (4/12; $33.3 \%$ ) (Table 3 ).

Although there was no growth from blood cultures, the isolation of Fusarium proliferatum from skin and nasal biopsy specimens in some severely immunocompromised patients with hematological malignancy $(n=3)$, aplastic anemia $(n=$ $1)$, and chronic renal failure $(n=1)$ underlined the predominance of the FFSC in deep invasive diseases. In one aplastic anemia and in two cancer patients who had symptoms of respiratory tract infection, F. proliferatum was grown from respiratory specimens like bronchoalveolar lavage, pleural fluid, and sputum. The FSSC caused localized infections in four patients. Three of them suffered from hematological malignancy, while in a patient with larynx cancer, F. solani s.s. (FSSC-5) was obtained from three different sputum specimens. One isolate of $F$. dimerum (FDSC) and one of $F$. oxysporum (FOSC) grew from skin biopsies and sputum samples in patients with paraplegia and hepatic cirrhosis, respectively.

The FSSC was responsible for most $(6 / 7 ; 85.7 \%)$ of the superficial infections (Table 3): Fusarium keratoplasticum was isolated from two onychomycosis cases, and $F$. solani s.s. (FSSC-5) was isolated from nail, corneal, and skin scrapings in patients with onychomycosis (one patient), keratitis (two patients), and diabetes mellitus (one patient), respectively.

Antifungal susceptibility tests were performed for 32 strains (isolated from 33 patients) which were identified by MLST. The overall results obtained from visual and/or spectrophotometric readings were similar for the MIC endpoints after 48 and $72 \mathrm{~h}$ of incubation. The GM values of MICs (mg/ L) across all isolates used in this study were as follows (in increasing order): amphotericin $\mathrm{B}, 0.84 \mathrm{mg} / \mathrm{L}$; voriconazole,
Table 1 Underlying diseases of patients with Fusarium infections

\begin{tabular}{|c|c|c|c|}
\hline \multirow[t]{2}{*}{ Underlying disease (no. of patients) } & \multicolumn{3}{|c|}{ Fusarium infections, no. of patients } \\
\hline & Superficial & Locally invasive & Disseminated \\
\hline \multicolumn{2}{|l|}{ Hematological malignancy $^{\mathrm{a}}(19)$} & 7 & 12 \\
\hline \multicolumn{2}{|l|}{ Solid organ carcinoma ${ }^{\mathrm{b}}(5)$} & 4 & 1 \\
\hline Chronic disorder $^{\mathrm{c}}(12)$ & 2 & 9 & 1 \\
\hline No underlying disease; keratitis (4) & \multicolumn{3}{|l|}{4} \\
\hline No underlying disease; onychomycosis (4) & \multicolumn{3}{|l|}{4} \\
\hline Other (3) & $1^{\mathrm{d}}$ & $1^{\mathrm{e}}$ & $1^{\mathrm{f}}$ \\
\hline Total (47) & 11 & 21 & 15 \\
\hline \multicolumn{4}{|c|}{$\begin{array}{l}\text { a Acute myeloid leukemia (11), acute lymphoblastic leukemia (5), multiple myeloma (1), myelodysplastic syn- } \\
\text { drome (1), T-cell lymphoma (1) }\end{array}$} \\
\hline \multicolumn{4}{|c|}{${ }^{\mathrm{b}}$ Larynx cancer (1), lung cancer (1), malign melanoma (1), adrenal cortex cancer (1), pancreas cancer (1) } \\
\hline \multicolumn{4}{|c|}{$\begin{array}{l}\text { 'Peripheral artery disease (2), aplastic anemia (2), Wegener's granulomatosis (1), cerebrovascular disease (1), } \\
\text { renal transplant (1), chronic renal failure (1), hepatic cirrhosis (1), paraplegia (1), chronic obstructive pulmonary } \\
\text { disease (1), diabetes (1) }\end{array}$} \\
\hline \multicolumn{4}{|l|}{${ }^{\mathrm{d}}$ Burn } \\
\hline \multicolumn{4}{|l|}{${ }^{\mathrm{e}}$ Hydatid cyst } \\
\hline \multicolumn{4}{|l|}{${ }^{\mathrm{f}}$ Low-birth-weight newborn } \\
\hline
\end{tabular}


Table 2 List of molecularly identified Fusarium isolates during the period 1995-2014

\begin{tabular}{|c|c|c|c|c|c|c|c|}
\hline Patient & Specimen & $\begin{array}{l}\text { Isolation } \\
\text { date }\end{array}$ & Infection & Underlying disease & $\begin{array}{l}\text { CBS } \\
\text { number }\end{array}$ & $\begin{array}{l}\text { Alternative } \\
\text { number }\end{array}$ & Fusarium species \\
\hline Patient 1 & Blood & 09-03-2010 & $\mathrm{D}$ & Acute myeloid leukemia & 139006 & 28623 & Fusarium petroliphilum (FSSC) \\
\hline Patient 2 & Nasal biopsy & 26-04-2013 & LI & Myelodysplastic syndrome & 138932 & 40009 & Fusarium petroliphilum (FSSC) \\
\hline \multirow[t]{4}{*}{ Patient 3} & Blood & $16-07-2013$ & $\mathrm{D}$ & Acute lymphoblastic leukemia & 139011 & 40686 & Fusarium petroliphilum (FSSC) \\
\hline & Nasal biopsy & 08-07-2013 & & & 139009 & 40600 & Fusarium petroliphilum (FSSC) \\
\hline & Skin biopsy & $12-07-2013$ & & & 139010 & 40625 & Fusarium petroliphilum (FSSC) \\
\hline & Skin biopsy & $12-07-2013$ & & & 139019 & 40654 & Fusarium petroliphilum (FSSC) \\
\hline Patient 4 & Blood & $12-05-2014$ & $\mathrm{D}$ & Acute myeloid leukemia & 139324 & 43248 & Fusarium petroliphilum (FSSC) \\
\hline Patient 5 & Blood & $20-05-2014$ & $\mathrm{D}$ & Acute myeloid leukemia & 139013 & 43325 & Fusarium petroliphilum (FSSC) \\
\hline Patient 6 & Nail scraping & 02-01-2008 & $\mathrm{S}$ & Onychomycosis & 139016 & 22840 & Fusarium solani s.s. (FSSC-5) \\
\hline \multirow[t]{3}{*}{ Patient 7} & Sputum & 04-03-2008 & LI & Larynx cancer & 138927 & 23239 & Fusarium solani s.s. (FSSC-5) \\
\hline & Sputum & 05-03-2008 & & & 139205 & 23250 & Fusarium solani s.s. (FSSC-5) \\
\hline & Sputum & $11-03-2008$ & & & 138999 & 23292 & Fusarium solani s.s. (FSSC-5) \\
\hline Patient 8 & Skin scraping & $16-05-2012$ & $\mathrm{~S}$ & Diabetes & 139007 & 36403 & Fusarium solani s.s. (FSSC-5) \\
\hline Patient 9 & Nasal biopsy & $31-05-2013$ & LI & Acute myeloid leukemia & 139008 & 40273 & Fusarium solani s.s. (FSSC-5) \\
\hline Patient 10 & Cornea scraping & $15-11-2013$ & $\mathrm{~S}$ & Keratitis & 139012 & 41672 & Fusarium solani s.s. (FSSC-5) \\
\hline \multirow[t]{2}{*}{ Patient 11} & Cornea scraping & 04-08-2014 & $\mathrm{S}$ & Keratitis & 139199 & 43937 & Fusarium solani s.s. (FSSC-5) \\
\hline & Cornea scraping & 08-08-2014 & & & 139200 & 43976 & Fusarium solani s.s. (FSSC-5) \\
\hline Patient 12 & Nail scraping & $05-02-2010$ & $\mathrm{~S}$ & Onychomycosis & 139005 & 28341 & Fusarium keratoplasticum (FSSC) \\
\hline Patient 13 & Nail scraping & $13-07-2012$ & $\mathrm{~S}$ & Onychomycosis & 139017 & 37057 & Fusarium keratoplasticum (FSSC) \\
\hline Patient 14 & Skin biopsy & $14-12-2009$ & LI & Acute myeloid leukemia & 139197 & 27854 & Fusarium solani s.s. (FSSC-6) \\
\hline Patient 15 & Blood & $26-07-2000$ & $\mathrm{D}$ & Acute lymphoblastic leukemia & 138998 & 7810 & Fusarium proliferatum (FFSC) \\
\hline Patient 16 & Nasal biopsy & 24-08-2004 & LI & Acute myeloid leukemia & 138924 & 14663 & Fusarium proliferatum (FFSC) \\
\hline Patient 17 & Skin biopsy & $01-12-2005$ & LI & Chronic renal failure & 138925 & 17857 & Fusarium proliferatum (FFSC) \\
\hline Patient 18 & BAL & 02-05-2008 & LI & Aplastic anemia & 139000 & 23660 & Fusarium proliferatum (FFSC) \\
\hline Patient 19 & Skin biopsy & $07-05-2008$ & LI & Acute myeloid leukemia & 139001 & 23690 & Fusarium proliferatum (FFSC) \\
\hline Patient 20 & Blood & $11-08-2008$ & $\mathrm{D}$ & Acute lymphoblastic leukemia & 139003 & 24321 & Fusarium proliferatum (FFSC) \\
\hline Patient 21 & Sputum & $06-11-2008$ & LI & Lung cancer & 139004 & 24861 & Fusarium proliferatum (FFSC) \\
\hline Patient 22 & Cornea scraping & $16-01-2012$ & $\mathrm{~S}$ & Keratitis & 138929 & 35142 & Fusarium proliferatum (FFSC) \\
\hline \multirow[t]{2}{*}{ Patient 23} & Nasal biopsy & $16-05-2012$ & LI & Aplastic anemia & 138930 & 36392 & Fusarium proliferatum (FFSC) \\
\hline & Nasal biopsy & $22-05-2012$ & & & 139203 & 36465 & Fusarium proliferatum (FFSC) \\
\hline Patient 24 & Blood & $14-06-2012$ & $\mathrm{D}$ & Malign melanoma & 138928 & 29541 & Fusarium proliferatum (FFSC) \\
\hline Patient 25 & Nasal biopsy & 05-07-2013 & LI & Acute myeloid leukemia & 139198 & 40572 & Fusarium proliferatum (FFSC) \\
\hline \multirow[t]{4}{*}{ Patient 26} & Blood & 03-06-2014 & $\mathrm{D}$ & Acute lymphoblastic leukemia & 139014 & 43498 & Fusarium proliferatum (FFSC) \\
\hline & Nasal biopsy & 09-05-2014 & & & 138933 & 43242 & Fusarium proliferatum (FFSC) \\
\hline & Blood & 05-06-2014 & & & 138934 & 43445 & Fusarium proliferatum (FFSC) \\
\hline & Blood & 09-06-2014 & & & 139201 & 43474 & Fusarium proliferatum (FFSC) \\
\hline Patient 27 & Pleural fluid & 28-11-2014 & LI & Pancreas cancer & 139325 & 45004 & Fusarium proliferatum (FFSC) \\
\hline Patient 28 & Blood & $30-10-2007$ & $\mathrm{D}$ & Acute myeloid leukemia & 139015 & 22525 & Fusarium verticillioides (FFSC) \\
\hline Patient 29 & Blood & $17-09-2012$ & $\mathrm{D}$ & T-cell lymphoma & 139018 & 37791 & Fusarium verticillioides (FFSC) \\
\hline \multirow[t]{2}{*}{ Patient 30} & Blood & 21-10-2014 & $\mathrm{D}$ & Acute lymphoblastic leukemia & 139202 & 44597 & Fusarium verticillioides (FFSC) \\
\hline & Skin biopsy & $13-10-2014$ & & & 139204 & 44536 & Fusarium verticillioides (FFSC) \\
\hline \multirow[t]{2}{*}{ Patient 31} & Blood & $07-12-2012$ & $\mathrm{D}$ & Acute myeloid leukemia & 138931 & 38603 & Fusarium andiyazi (FFSC) \\
\hline & Blood & $11-12-2012$ & & & 139195 & 38621 & Fusarium andiyazi (FFSC) \\
\hline Patient 32 & Sputum & $20-06-2007$ & LI & Hepatic cirrhosis & 138926 & 21689 & Fusarium oxysporum (FOSC) \\
\hline \multirow[t]{2}{*}{ Patient 33} & Skin biopsy & 01-08-2008 & LI & Paraplegia & 139196 & 24262 & Fusarium dimerum (FDSC) \\
\hline & Skin biopsy & 01-08-2008 & & & 139002 & 24264 & Fusarium dimerum (FDSC) \\
\hline
\end{tabular}

$B A L$ bronchoalveolar lavage; $D$ disseminated; $L I$ locally invasive; $S$ superficial 
Table 3 Fusarium species in different types of infections

\begin{tabular}{|c|c|c|c|c|}
\hline \multirow[t]{2}{*}{ Species } & \multicolumn{4}{|c|}{ Fusarium infections, no. of patients } \\
\hline & Superficial & Locally invasive & Disseminated & Total \\
\hline F. petroliphilum (FSSC) & & 1 & 4 & 5 \\
\hline F. solani s.s. (FSSC-5) & 4 & 2 & & 6 \\
\hline F. keratoplasticum (FSSC) & 2 & & & 2 \\
\hline F. solani s.s. (FSSC-6) & & 1 & & 1 \\
\hline F. proliferatum (FFSC) & 1 & 8 & 4 & 13 \\
\hline F. verticillioides (FFSC) & & & 3 & 3 \\
\hline F. andiyazi (FFSC) & & & 1 & 1 \\
\hline F. oxysporum (FOSC) & & 1 & & 1 \\
\hline F. dimerum (FDSC) & & 1 & & 1 \\
\hline Total & 7 & 14 & 12 & 33 \\
\hline
\end{tabular}

$3.83 \mathrm{mg} / \mathrm{L}$; posaconazole, $5.3 \mathrm{mg} / \mathrm{L}$; caspofungin, $15.66 \mathrm{mg} /$ $\mathrm{L}$; anidulafungin, $>16 \mathrm{mg} / \mathrm{L}$; itraconazole, $59.97 \mathrm{mg} / \mathrm{L}$; fluconazole, $61.29 \mathrm{mg} / \mathrm{L}$; and flucytosine, $>64 \mathrm{mg} / \mathrm{L}$ (Table 4). Of note, all of the species showed high MIC/MEC values for flucytosine, fluconazole, itraconazole, anidulafungin, and caspofungin.

Amphotericin B had the highest in vitro activity (ranging from 0.125 to $4 \mathrm{mg} / \mathrm{L}$ ) against all species, with the MIC ranging from 0.125 to $4 \mathrm{mg} / \mathrm{L}$. However, both voriconazole and posaconazole showed interspecies variability across and within Fusarium species complexes. The members of the FSSC (MIC ranging from 2 to $8 \mathrm{mg} / \mathrm{L}$ ) and especially F. petroliphilum strains (MIC ranging from 8 to $16 \mathrm{mg} / \mathrm{L}$ ) showed higher MIC values than other species for voriconazole. A similar pattern of susceptibility was also observed with posaconazole against the FSSC (MIC ranging from 0.125 to $>16 \mathrm{mg} / \mathrm{L}$ ).

\section{Discussion}

In the present study, cases of fusariosis occurring in a tertiarycare university hospital in Turkey over the last 20 years were evaluated. Both species distribution and antifungal susceptibility of the isolates were determined. These are the first epidemiological data on fusarioses from Turkey. Similar to recent surveys elsewhere, a statistically significant increase in the incidence of fusarioses over the years was also determined in this study [26, 27].

Fusarium species cause a broad spectrum of infections in humans, including superficial, locally invasive, and disseminated infections $[13,14]$. Clinical manifestations largely depend on the immune status of the host and the portal of entry, which include paranasal sinuses, lungs, and skin [13]. Among immunocompetent hosts, keratitis and onychomycosis are the most common infections and, occasionally, mycetoma by

Table 4 Geometric means (GMs) of the minimum inhibitory concentrations (MICs)/minimum effective concentrations (MECs) and MIC/MEC ranges obtained by testing the susceptibility of 32 Fusarium strains to antifungal agents

\begin{tabular}{|c|c|c|c|c|c|c|c|c|c|c|c|c|c|c|c|c|}
\hline \multirow[b]{3}{*}{ Species $(\mathrm{n})$} & \multicolumn{16}{|c|}{ MIC/MEC (mg/L) } \\
\hline & \multicolumn{2}{|c|}{ Amphotericin B } & \multicolumn{2}{|c|}{ Voriconazole } & \multicolumn{2}{|c|}{ Posaconazole } & \multicolumn{2}{|c|}{ Fluconazole } & \multicolumn{2}{|c|}{ Itraconazole } & \multicolumn{2}{|c|}{ Anidulafungin } & \multicolumn{2}{|c|}{ Caspofungin } & \multicolumn{2}{|c|}{$\begin{array}{l}\text { 5- } \\
\text { Fluorocytosine }\end{array}$} \\
\hline & $\begin{array}{l}\text { MIC } \\
\text { Range }\end{array}$ & $\begin{array}{l}\text { GM } \\
\text { MIC }\end{array}$ & $\begin{array}{l}\text { MIC } \\
\text { Range }\end{array}$ & $\begin{array}{l}\text { GM } \\
\text { MIC }\end{array}$ & $\begin{array}{l}\text { MIC } \\
\text { Range }\end{array}$ & $\begin{array}{l}\text { GM } \\
\text { MIC }\end{array}$ & $\begin{array}{l}\text { MIC } \\
\text { Range }\end{array}$ & $\begin{array}{l}\text { GM } \\
\text { MIC }\end{array}$ & $\begin{array}{l}\text { MIC } \\
\text { Range }\end{array}$ & $\begin{array}{l}\text { GM } \\
\text { MIC }\end{array}$ & $\begin{array}{l}\text { MEC } \\
\text { Range }\end{array}$ & $\begin{array}{l}\text { GM } \\
\text { MEC }\end{array}$ & $\begin{array}{l}\text { MEC } \\
\text { Range }\end{array}$ & $\begin{array}{l}\text { GM } \\
\text { MEC }\end{array}$ & $\begin{array}{l}\text { MIC } \\
\text { Range }\end{array}$ & $\begin{array}{l}\text { GM } \\
\text { MIC }\end{array}$ \\
\hline FSSC (14) & $0.25-2$ & 0.86 & $2-16$ & 5.94 & $0.12->16$ & 11.28 & $>64$ & $>64$ & $>64$ & $>64$ & $>16$ & $>16$ & $>16$ & $>16$ & $>64$ & $>64$ \\
\hline Fusarium petroliphilum (5) & $0.25-1$ & 0.57 & $8-16$ & 9.19 & $>16$ & $>16$ & $>64$ & $>64$ & $>64$ & $>64$ & $>16$ & $>16$ & $>16$ & $>16$ & $>64$ & $>64$ \\
\hline Fusarium solani s.s (FSSC-5) (6) & $0.25-1$ & 0.79 & $2-8$ & 4.49 & $>16$ & $>16$ & $>64$ & $>64$ & $>64$ & $>64$ & $>16$ & $>16$ & $>16$ & $>16$ & $>64$ & $>64$ \\
\hline Fusarium keratoplasticum (2) & 2 & 2 & 8 & 8 & $0.12-16$ & 1.41 & $>64$ & $>64$ & $>64$ & $>64$ & $>16$ & $>16$ & $>16$ & $>16$ & $>64$ & $>64$ \\
\hline Fusarium solani s.s. (FSSC-6) (1) & 2 & 2 & 2 & 2 & $>16$ & $>16$ & $>64$ & $>64$ & $>64$ & $>64$ & $>16$ & $>16$ & $>16$ & $>16$ & $>64$ & $>64$ \\
\hline FFSC (16) & $0.12-8$ & 0.88 & $1-8$ & 2.71 & $0.12-16$ & 2.36 & $16-64$ & 58.69 & $8-64$ & 56.20 & $>16$ & $>16$ & $8-16$ & 15.32 & $>64$ & $>64$ \\
\hline Fusarium proliferatum (12) & $0.12-1$ & 0.56 & $1-8$ & 3.56 & $0.12-16$ & 5.02 & $>64$ & $>64$ & $>64$ & $>64$ & $>16$ & $>16$ & $>16$ & $>16$ & $>64$ & $>64$ \\
\hline Fusarium verticillioides (3) & $2-4$ & 2.52 & 1 & 1 & $0.12-0.25$ & 0.16 & $>64$ & $>64$ & $>64$ & $>64$ & $>16$ & $>16$ & $>16$ & $>16$ & $>64$ & $>64$ \\
\hline Fusarium andiyazi (1) & 8 & 8 & 2 & 2 & 1 & 1 & 16 & 16 & 8 & 8 & $-^{\mathrm{a}}$ & $-^{\mathrm{a}}$ & 8 & 8 & $\mathrm{-a}^{\mathrm{a}}$ & $-^{\mathrm{a}}$ \\
\hline FOSC (1) Fusarium oxysporum & 0.5 & 0.5 & 2 & 2 & 16 & 16 & $>64$ & $>64$ & $>64$ & $>64$ & $>16$ & $>16$ & $>16$ & $>16$ & $>64$ & $>64$ \\
\hline FDSC (1) Fusarium dimerum & 0.5 & 0.5 & 4 & 4 & $>16$ & $>16$ & $>64$ & $>64$ & $>64$ & $>64$ & $>16$ & $>16$ & $>16$ & $>16$ & $>64$ & $>64$ \\
\hline TOTAL (32) & $0.12-8$ & 0.84 & $1-16$ & 3.83 & $0.12-16$ & 5.30 & $16->64$ & 61,29 & $8->64$ & 59.97 & $>16$ & $>16$ & $8->16$ & 15,66 & $>64$ & $>64$ \\
\hline
\end{tabular}

${ }^{\mathrm{a}}$ Not examined; MIC: minimum inhibitory concentration; MEC: minimum effective concentration

\begin{tabular}{|c|c|c|c|c|c|}
\hline Color code & $\leq 1 \mathrm{mg} / \mathrm{l}$ & $2 \mathrm{mg} / 1$ & $4 \mathrm{mg} / \mathrm{l}$ & $8 \mathrm{mg} / \mathrm{l}$ & $\geq 16 \mathrm{mg} / \mathrm{l}$ \\
\hline
\end{tabular}


Fusarium spp. is observed. Unlike infections in the normal host, fusariosis in the immunocompromised population is typically invasive and disseminated. Disseminated infections occur mainly in patients with hematological malignancies and have also occasionally been reported in the immunocompromised patients due to transplantation, solid tumors, and autoimmune disorders $[13,27-30]$. In line with this, $80 \%$ of the patients with disseminated fusariosis in the present study had hematological malignancy. The remaining patients with disseminated infections were also severely immunocompromised, e.g., due to solid tumor, autoimmune disease, or low birth weight.

The principal portal of entry for Fusarium species seems to be the airways or traumatic inoculation. Sinus and lung involvement is common and these foci may serve as sites for dissemination in immunocompromised hosts. Clinical manifestations of fusarial sinusitis and pneumonia are indistinguishable from those caused by Aspergillus spp., but especially lung involvement is associated with higher mortality [13]. Involvement of the skin is another important manifestation of fusariosis and can represent a primary site of infection or a manifestation of secondary metastasis in patients with disseminated fusariosis. Among immunocompromised patients, skin lesions may be the single source of diagnosis [13, 31].

In this study, nearly half $(44.7 \%)$ of the cases were localized infections, while lung, sinuses, and skin involvement was seen in different underlying conditions, like hematological disorders, solid malignancies, and chronic illnesses. Definitive diagnosis of Fusarium species requires isolation of the agent from infected sites, and culture identification from biopsy samples is especially important because of the histopathological similarities between Fusarium and other hyalohyphomycetes agents. Clinicians should be aware of local infections that may occur during follow-up of high-risk patients, and different samples are of importance in detection.

In vitro, the genus Fusarium can be identified by the production of hyaline, crescent or banana-shaped, multicellular macroconidia. However, species identification is difficult and requires molecular methods [5, 18]. In this study, we used MLST for identification to the species level.

Globally, the FSSC is the most common group encountered in human infections and is present in approximately $50 \%$ of patients [13]. Being responsible for about $20 \%$ of infections, the FOSC ranks second [13]. In the present study, however, the FFSC was found to be the most frequently detected complex $(51.5 \%)$, followed by the FSSC (42.4\%), while the FOSC was isolated from only a single patient. The $F$. andiyazi strain isolated from one patient was the first isolation from a human case that we previously reported [32]. In a recent survey conducted in Europe (involving our region as well), the FFSC was also reported to be prevalent [31]. These data support the view that regional differences exist in the distribution of Fusarium species [10].

A link between species identity and course of infection has been observed in several studies. For example, the FFSC was prevalently isolated from invasive and disseminated infections $[8,10,17] . F$. proliferatum and $F$. verticillioides are the most commonly encountered human opportunists in this complex. Also, in our study, F. proliferatum was the predominant species in locally invasive infections and along with F. petroliphilum in disseminated infections [33]. The FSSC has also been reported as the most commonly isolated species complex in superficial infections, mainly keratitis and onychomycosis, in America and Asia, while the FOSC ranks first in such infections in Europe [9, 34-36]. Although the number of cases was low in our study, the FSSC was responsible in $85.7 \%$ of superficial infections.

In the present study, flucytosine, fluconazole, itraconazole, anidulafungin, and caspofungin were ineffective against all isolates. Species-specific differences in the susceptibility of Fusarium isolates was apparent in the MICs to amphotericin B, voriconazole, and posaconazole. In agreement with previous reports [16, 37], amphotericin $\mathrm{B}$ was the most effective agent across all isolates. The European Society of Clinical Microbiology and Infectious Diseases (ESCMID) and the European Confederation of Medical Mycology (ECMM) recommend amphotericin B and voriconazole as the first optional agents in the treatment of cases with fusariosis [18]. However, in the present study, these compounds were not effective against some of the species, sometimes indicating high degrees of intraspecific variability (Table 4). Some studies have reported that the MIC values in $F$. verticillioides were high for amphotericin B and low for posaconazole [16, 37]. Similarly, we also observed activity of posaconazole against this species, but with a limited degree of confidence.

In conclusion, our study indicated that regional differences exist in the distribution of Fusarium species and that species-specific differences might also reflect in antifungal susceptibility patterns. As the observed number of fusarioses, particularly disseminated cases, are increasing, monitoring of local epidemiological data by determining fungal identity and antifungal susceptibility testing will definitely help clinicians develop appropriate therapies that have a high probability of successfully treating disseminated fusariosis.

Acknowledgments Burcu Dalyan Cilo was supported by grants from the Scientific and Technological Research Council of Turkey and an FEMS fellowship via the affiliates Turkish Microbiological Society. Abdullah Mohammed Said Al-Hatmi was financially supported by the Ministry of Health, Muscat, Oman.

Conflict of interest The authors declare to have no conflict of interest. 


\section{References}

1. Leslie JF, Summerell BA (2006) The Fusarium laboratory manual. Blackwell, London

2. O'Donnell K, Sutton DA, Fothergill A, McCarthy D, Rinaldi MG, Brandt ME et al (2008) Molecular phylogenetic diversity, multilocus haplotype nomenclature, and in vitro antifungal resistance within the Fusarium solani species complex. J Clin Microbiol 46:2477-2490

3. O'Donnell K, Gueidan C, Sink S, Johnston PR, Crous PW, Glenn A et al (2009) A two-locus DNA sequence database for typing plant and human pathogens within the Fusarium oxysporum species complex. Fungal Genet Biol 46:936-948

4. O'Donnell K, Sutton DA, Rinaldi MG, Gueidan C, Crous PW, Geiser DM (2009) Novel multilocus sequence typing scheme reveals high genetic diversity of human pathogenic members of the Fusarium incarnatum-F. equiseti and $F$. chlamydosporum species complexes within the United States. J Clin Microbiol 47:38513861

5. O'Donnell K, Sutton DA, Rinaldi MG, Sarver BA, Balajee SA, Schroers HJ et al (2010) Internet-accessible DNA sequence database for identifying fusaria from human and animal infections. J Clin Microbiol 48:3708-3718

6. Geiser DM, Aoki T, Bacon CW, Baker SE, Bhattacharyya MK, Brandt ME et al (2013) One fungus, one name: defining the genus Fusarium in a scientifically robust way that preserves longstanding use. Phytopathology 103:400-408

7. de Hoog GS, Haase G, Chaturvedi V, Walsh TJ, Meyer W, Lackner M (2013) Taxonomy of medically important fungi in the molecular era. Lancet Infect Dis 13:385-386

8. Lortholary O, Obenga G, Biswas P, Caillot D, Chachaty E, Bienvenu AL et al (2010) International retrospective analysis of 73 cases of invasive fusariosis treated with voriconazole. Antimicrob Agents Chemother 54:4446-4450

9. Migheli Q, Balmas V, Harak H, Sanna S, Scherm B, Aoki T et al (2010) Molecular phylogenetic diversity of dermatologic and other human pathogenic fusarial isolates from hospitals in northern and central Italy. J Clin Microbiol 48:1076-1084

10. van Diepeningen AD, Feng P, Ahmed S, Sudhadham M, Bunyaratavej S, de Hoog GS (2015) Spectrum of Fusarium infections in tropical dermatology evidenced by multilocus sequencing typing diagnostics. Mycoses 58(1):48-57

11. Kredics L, Narendran V, Shobana CS, Vágvölgyi C, Manikandan P; Indo-Hungarian Fungal Keratitis Working Group (2015) Filamentous fungal infections of the cornea: a global overview of epidemiology and drug sensitivity. Mycoses 58:243-260

12. Avelino-Silva VI, Ramos JF, Leal FE, Testagrossa L, Novis YS (2015) Disseminated Fusarium infection in autologous stem cell transplant recipient. Braz J Infect Dis 19:90-93

13. Nucci M, Anaissie E (2007) Fusarium infections in immunocompromised patients. Clin Microbiol Rev 20:695-704

14. van Diepeningen $\mathrm{AD}$, Al-Hatmi AMS, Brankovics B, de Hoog GS (2014) Taxonomy and clinical spectra of Fusarium species: where do we stand in 2014? Curr Clin Microbiol Rep 1:10-18

15. Nucci M, Marr KA, Vehreschild MJGT, de Souza CA, Velasco E, Cappellano $P$ et al (2014) Improvement in the outcome of invasive fusariosis in the last decade. Clin Microbiol Infect 20:580-585

16. Azor M, Gené J, Cano J, Guarro J (2007) Universal in vitro antifungal resistance of genetic clades of the Fusarium solani species complex. Antimicrob Agents Chemother 51:1500-1503

17. Al-Hatmi AMS, van Diepeningen AD, Curfs-Breuker I, de Hoog GS, Meis JF (2015) Specific antifungal susceptibility profiles of opportunists in the Fusarium fujikuroi complex. J Antimicrob Chemother 70:1068-1071. doi:10.1093/jac/dku505
18. Tortorano AM, Richardson M, Roilides E, van Diepeningen A, Caira M, Munoz P et al (2014) ESCMID and ECMM joint guidelines on diagnosis and management of hyalohyphomycosis: Fusarium spp., Scedosporium spp. and others. Clin Microbiol Infect 20(Suppl 3):27-46

19. Alastruey-Izquierdo A, Cuenca-Estrella M, Monzón A, Mellado E, Rodríguez-Tudela JL (2008) Antifungal susceptibility profile of clinical Fusarium spp. Isolates identified by molecular methods. J Antimicrob Chemother 61:805-809

20. Möller EM, Bahnweg G, Sandermann H, Geiger HH (1992) A simple and efficient protocol for isolation of high molecular weight DNA from filamentous fungi, fruit bodies, and infected plant tissues. Nucleic Acids Res 20:6115-6116

21. White TJ, Bruns TD, Lee SB, Taylor JW (1990) Amplification and direct sequencing of fungal ribosomal RNA genes for phylogenetics. In: Innis MA, Gelfand DH, Sninsky JJ, White TJ (eds) PCR protocols: a guide to methods and applications. Academic Press, New York, pp 315-322

22. Geiser DM, del Mar Jiménez-Gasco M, Kang S, Makalowska I, Veeraraghavan N, Ward TJ et al (2004) FUSARIUM-ID v. 1.0: a DNA sequence database for identifying Fusarium. Eur J Plant Pathol 110:473-479

23. Schroers HJ, O’Donnell K, Lamprecht SC, Kammeyer PL, Johnson S, Sutton DA et al (2009) Taxonomy and phylogeny of the Fusarium dimerum species group. Mycologia 101:44-70

24. Clinical and Laboratory Standards Institute (CLSI) (2008) Reference method for broth dilution antifungal susceptibility testing of filamentous fungi; Approved standard-Second edition. CLSI document M38-A2. CLSI, Wayne

25. Mansur AT, Artunkal S, Ener B (2011) Fusarium oxysporum infection of stasis ulcer: eradication with measures aimed to improve stasis. Mycoses 54:e205-e207

26. Pagano L, Caira M, Candoni A, Offidani M, Fianchi L, Martino B et al (2006) The epidemiology of fungal infections in patients with hematologic malignancies: the SEIFEM-2004 study. Haematologica 91:1068-1075

27. Nucci M, Varon AG, Garnica M, Akiti T, Barreiros G, Trope $\mathrm{BM}$ et al (2013) Increased incidence of invasive fusariosis with cutaneous portal of entry, Brazil. Emerg Infect Dis 19: $1567-1572$

28. Guinvarc'h A, Guilbert L, Marmorat-Khuong A, Lavarde V, Chevalier P, Amrein C et al (1998) Disseminated Fusarium solani infection with endocarditis in a lung transplant recipient. Mycoses 41:59-61

29. Park BJ, Pappas PG, Wannemuehler KA, Alexander BD, Anaissie EJ, Andes DR et al (2011) Invasive non-Aspergillus mold infections in transplant recipients, United States, 2001-2006. Emerg Infect Dis 17:1855-1864

30. Edupuganti S, Rouphael N, Mehta A, Eaton M, Heller JG, Bressler A et al (2011) Fusarium falciforme vertebral abscess and osteomyelitis: case report and molecular classification. J Clin Microbiol 49: 2350-2353

31. Tortorano AM, Prigitano A, Esposto MC, Arsic Arsenijevic V, Kolarovic J, Ivanovic D et al (2014) European Confederation of Medical Mycology (ECMM) epidemiological survey on invasive infections due to Fusarium species in Europe. Eur J Clin Microbiol Infect Dis 33:1623-1630

32. Kebabcı N, van Diepeningen AD, Ener B, Ersal T, Meijer M, AlHatmi AMS et al (2014) Fatal breakthrough infection with Fusarium andiyazi: new multi-resistant aetiological agent crossreacting with Aspergillus galactomannan enzyme immunoassay. Mycoses 57:249-255

33. Ersal T, Al-Hatmi AMS, Cilo BD, Curfs-Breuker I, Meis JF, Özkalemkaș F et al (2015) Fatal disseminated infection with Fusarium petroliphilum. Mycopathologia 179:119-124 
34. Chi CC, Wang SH, Chou MC (2005) The causative pathogens of onychomycosis in southern Taiwan. Mycoses 48:413-420

35. Castro López N, Casas C, Sopo L, Rojas A, Del Portillo P, Cepero de García MC et al (2009) Fusarium species detected in onychomycosis in Colombia. Mycoses 52:350-356
36. Ninet B, Jan I, Bontems O, Léchenne B, Jousson O, Lew D et al (2005) Molecular identification of Fusarium species in onychomycoses. Dermatology 210:21-25

37. Muhammed M, Coleman JJ, Carneiro HA, Mylonakis E (2011) The challenge of managing fusariosis. Virulence 2:91-96 